\title{
Longterm Safety and Efficacy of Subcutaneous Abatacept in Patients with Rheumatoid Arthritis: 5-year Results from a Phase IIIb Trial
}

\author{
Mark C. Genovese, César Pacheco-Tena, Arturo Covarrubias, Gustavo Leon, Eduardo Mysler, \\ Mauro Keiserman, Robert M. Valente, Peter Nash, J. Abraham Simon-Campos, Jane Box, \\ Clarence W. Legerton III, Evgeny Nasonov, Patrick Durez, Ayanbola Elegbe, Robert Wong, \\ Xiaohui Li, Subhashis Banerjee, and Rieke Alten
}

\begin{abstract}
Objective. To assess 5-year safety, tolerability, and efficacy of subcutaneous (SC) abatacept (ABA) in methotrexate (MTX)-refractory patients with rheumatoid arthritis (RA).

Methods. The Abatacept Comparison of sub[QU]cutaneous versus intravenous in Inadequate Responders to methotrexatE (ACQUIRE) phase IIIb, randomized, double-dummy, multinational trial compared efficacy and safety of SC and intravenous (IV) ABA in patients with RA. In the initial 6-month double-blind (DB) period, patients received IV or SC ABA, plus MTX, and in the subsequent open-label longterm extension (LTE) period, all patients received SC ABA (125 mg/wk). The final 5-year safety, tolerability, and efficacy analyses are reported.

Results. Of 1385 patients who completed the DB period, 1372 entered LTE and 945 (68.8\%) completed $\geq 5$ years of treatment. During LTE, 97 (7.1\%) patients discontinued treatment because of an adverse event (AE). Incidence rate (IR; event/100 patient-yrs of exposure; based on LTE data, 95\% CI) for $\mathrm{AE}$ of interest were the following: serious $\mathrm{AE} 7.73$ (6.96-8.58), infection 38.60 (36.24-41.12), serious infection 1.68 (1.35-2.07), malignancies 1.09 (0.84-1.42), and autoimmune disorders 1.33 (1.05-1.69), and were stable over time. No association between immunogenicity and either worsening of ABA safety or loss of efficacy was noted. Efficacy in the LTE was consistent with the DB period and was maintained to the end of the study.

Conclusion. These 5-year data establish that SC ABA (125 mg/wk) has a consistent safety profile and durable efficacy for longterm treatment of patients with RA who had an inadequate response to MTX. (First Release April 15 2018; J Rheumatol 2018;45:1085-92; doi:10.3899/jrheum.170344)
\end{abstract}

Key Indexing Terms:

RHEUMATOID ARTHRITIS

DISEASE ACTIVITY

\section{CLINICAL TRIAL \\ C-REACTIVE PROTEIN}

From Stanford University, Palo Alto, California; Arthritis Center of Nebraska, Lincoln, Nebraska; Box Arthritis and Rheumatology of the Carolinas, Charlotte, North Carolina; Low Country Rheumatology, Charleston, South Carolina; Bristol-Myers Squibb, Princeton, New Jersey, USA; Universidad Autónoma de Chihuahua, Chihuahua; Unidad Reumatológica Las Américas S.C.P., Mérida; Köhler \& Milstein Research, Mérida, Mexico; Instituto De Ginecología Y Reproducción, Lima, Peru; Organización Médica de Investigación, Buenos Aires, Argentina; Pontifical Catholic University, Porto Alegre, Brazil; University of Queensland, Brisbane, Australia; Russian Academy of Medical Sciences, Moscow, Russia; Cliniques Universitaires Saint-Luc; Université Catholique de Louvain, Brussels, Belgium; Schlosspark-Klinik University Medicine, Berlin, Germany.

This study was sponsored by Bristol-Myers Squibb.

Dr. R.M. Valente received grant/research support from Novartis, BristolMyers Squibb, Pfizer, and Lilly. Dr. P. Nash received grant/research support, and speakers' bureau and consulting fees from Bristol-Myers Squibb. Dr. C.W. Legerton received grant/research support from AbbVie, Ablynx, Acerta, Amgen, AstraZeneca, Celgene, GSK, Janssen, Lilly, Bristol-Myers Squibb, Pfizer, Novartis, Sandoz, UCB Pharma, Daiichi Sankyo, ChemoCentryx, and Boehringer Ingelheim, and speakers' bureau fees from Celgene, Amgen, and Novartis. Dr. A. Elegbe, Dr. R. Wong, Dr. X. Li, and Dr. S. Banerjee are shareholders and employees of Bristol-Myers Squibb.

\begin{abstract}
M.C. Genovese, MD, Stanford University; C. Pacheco-Tena, MD, PhD Universidad Autónoma de Chihuahua; A. Covarrubias, MD, Unidad Reumatológica Las Américas S.C.P.; G. Leon, MD, Instituto De Ginecología Y Reproducción; E. Mysler, MD, Organización Médica de Investigación; M. Keiserman, MD, Pontifical Catholic University; R.M. Valente, MD, Arthritis Center of Nebraska; P. Nash, MBBS (Hons), FRACP, University of Queensland; J.A. Simon-Campos, PhD, Köhler \& Milstein Research; J. Box, MD, Box Arthritis and Rheumatology of the Carolinas; C.W. Legerton III, MD, Low Country Rheumatology; E. Nasonov, PhD, Russian Academy of Medical Sciences; P. Durez, MD, Cliniques Universitaires Saint-Luc, Université Catholique de Louvain; A. Elegbe, PhD, Bristol-Myers Squibb; R. Wong, MD, Bristol-Myers Squibb; X. Li, PhD, Bristol-Myers Squibb; S. Banerjee, MD, Bristol-Myers Squibb; R. Alten, $M D, P h D$, Schlosspark-Klinik University Medicine. Address correspondence to Dr. M.C. Genovese, Stanford University, Division of Immunology and Rheumatology, 1000 Welch Road \#203, Palo Alto, California 94304,USA.E-mail: genovese@stanford.edu

Full Release Article. For details, see Reprints and Permissions at jrheum.org Accepted for publication December 18, 2017.
\end{abstract}

Rheumatoid arthritis (RA) is a chronic, progressive, autoimmune condition that requires longstanding treatment

Personal non-commercial use only. The Journal of Rheumatology Copyright (c) 2018. All rights reserved. 
over the duration of the disease; hence, the monitoring and collection of longterm safety and efficacy data for RA therapies are imperative and can aid physician decisions on choice of therapeutic options. Patients with RA may have an increased risk of infection and some organ-specific malignancies ${ }^{1,2}$. Therefore, it is particularly important to monitor the incidence of infections and malignancies together with other adverse events (AE) in patients with RA treated with immunomodulatory agents.

Abatacept (ABA) is a selective $\mathrm{T}$ cell costimulation modulator available in both intravenous (IV) and subcutaneous (SC) formulations. IV ABA has been available in the United States (US) and Europe since 2005 and 2007, respectively, for the treatment of moderate to severe RA as monotherapy or concomitantly with disease-modifying antirheumatic drugs (DMARD). The SC formulation for patient self-administration was approved for use in RA in the US in 2011, in Europe in 2012, and other global regions thereafter. Longterm safety and efficacy of the IV formulation of ABA has been shown, with several studies demonstrating that both efficacy and safety were maintained up to 7 years in patients with an inadequate response to methotrexate $(\mathrm{MTX})^{3,4,5,6,7,8}$, and to 5 years in patients with an inadequate response to tumor necrosis factor inhibitors ${ }^{4}$. However, the longterm safety and efficacy of SC ABA are less well characterized $^{9,10,11,12}$. An integrated analysis of safety data from the double-blind (DB) and open-label periods of 5 clinical trials, including a total of 1879 patients with 4214.6 patient-years (PY) of exposure, showed that treatment with SC ABA (mean length of exposure $2.3 \mathrm{yrs}$ ) was associated with a low incidence of serious infections, malignancies, autoimmune events, and injection-site reactions ${ }^{12}$.

The Abatacept Comparison of sub[QU]cutaneous versus intravenous in Inadequate Responders to methotrexatE (ACQUIRE) trial was a phase IIIb, randomized, DB, double-dummy, multinational trial that compared the efficacy and safety of SC and IV ABA in patients with RA, and an inadequate response to $\geq 3$ months of $\mathrm{MTX}^{13}$. The study demonstrated comparable American College of Rheumatology $20 \%$ improvement (ACR20) criteria responses after 6 months and noninferiority of the SC to the IV formulation ${ }^{13}$. Following the 6-month (Day 189) DB period, outcomes were evaluated in an open-label, longterm extension (LTE) period, during which all patients received SC ABA 125 mg/week.

Here we report the final 5-year safety, tolerability, immunogenicity, and efficacy results of SC ABA 125 $\mathrm{mg} /$ week plus background MTX in the open-label LTE period of the ACQUIRE study.

\section{MATERIALS AND METHODS}

Study design and patient population. The ACQUIRE study (NCT00559585) design and inclusion/exclusion criteria have been published in detail ${ }^{10,13}$. Briefly, at study entry, patients were aged $\geq 18$ years with active RA, and had inadequate response to $\geq 3$ months of MTX treatment, $\geq 10$ swollen joints, $\geq 12$ tender joints, and C-reactive protein (CRP) levels $\geq 0.8 \mathrm{mg} / \mathrm{dl}$. In the DB period, patients were randomized (1:1) to receive SC ABA (125 $\mathrm{mg} / \mathrm{wk}$ ) on Day 1 (with addition of a single IV infusion of $10 \mathrm{mg} / \mathrm{kg}$ on Day 1) and weekly thereafter, or IV ABA (10 mg/kg) on days 1,15 , and 29 , and every 4 weeks thereafter ${ }^{10,13}$. Patients continued MTX at the same dose they were receiving at randomization ( $\mathrm{min} 15 \mathrm{mg} / \mathrm{wk}$, orally or parenterally as clinically indicated); low-dose stable oral corticosteroids $(<10 \mathrm{mg} / \mathrm{d}$ prednisone equivalent) and stable dose nonsteroidal antiinflammatory drugs (NSAID) were also permitted. The study was conducted in accordance with the Declaration of Helsinki and received Institutional Review Board/Independent Ethics Committee approval (research ethics board approval number 5136, Stanford University Panel on Human Subjects in Medical Research).

All patients who completed the 6-month (Day 169) DB period were eligible to enter the LTE period. In the open-label LTE period, all patients received SC ABA $125 \mathrm{mg} /$ week. Addition of conventional synthetic DMARD, but not biologics, and dose adjustments to MTX, corticosteroids, and NSAID were permitted. The LTE period continued for at least 5 years or when the SC formulation of ABA became commercially available in those countries, after which the study was terminated locally. The end of the study was defined as the final followup visit for the last patient (September 12 , 2014)

Assessments. Safety assessments were classified using the Medical Dictionary for Regulatory Activities (version 17.1) and performed at 12-week intervals during the LTE period, up to 168 days after the last dose date. All AE, serious (S-) AE, AE of special interest (including those associated with immunomodulatory drug use, such as infections, prespecified autoimmune disorders, malignancies, local injection-site reactions, and systemic injection reactions), vital signs, and laboratory test abnormalities were recorded. AE were included in the frequency calculations if they occurred while the patient was taking the study drug (during the LTE period), up to and including 56 days (equivalent to about $5 \times$ ABA half-life) after the last dose date.

Immunogenicity assessment was performed on serum samples collected prior to the administration of ABA, every 12-24 weeks throughout the LTE period and up to 168 days after the last dose. The presence of anti-ABA antibodies was evaluated using validated bridging electrochemiluminescence (Meso-Scale Discovery) that differentiated between the 2 antibody specificities: immunoglobulin (Ig) and/or junction region, and T cell lymphocyte costimulation inhibitors (CTLA-4) and possibly Ig. An antidrug-antibodypositive response was defined as a laboratory-reported titer value $\geq 10$ and further specified as being CTLA-4 and possibly Ig, and Ig and/or junction region-positive if baseline measurement was negative, missing, or less than postbaseline value. Samples that were positive for CTLA-4 and possibly Ig antibody were also tested for the presence of neutralizing ability when sufficient sample was available, and the ABA concentration was $<1 \mu \mathrm{g} / \mathrm{ml}$. Persistent immunogenicity response was defined as $\geq 2$ consecutive positives for the same reactivity occurring at least 12 weeks apart.

Efficacy assessments performed at 12-week and yearly intervals during the LTE period included ACR20, 50, and 70 improvement criteria, 28-joint count Disease Activity Score (DAS28)-defined low disease activity score (DAS28-CRP $\leq 3.2$ ), DAS-defined remission (DAS28-CRP $<2.6$ ), and physical function response [Health Assessment Questionnaire-Disability Index (HAQ-DI) improvement of $\geq 0.3$ units from baseline]. Additional patient-reported outcomes such as joint pain and global assessment of disease activity (assessed using a 0-100 visual analog scale) were also evaluated.

Serum ABA concentrations were determined using ELISA at 12- and 24-week intervals up to Day 729 of the LTE period.

Statistical analyses. Baseline demographic data and clinical characteristics were analyzed descriptively for all patients in the study. Safety and efficacy analyses included all patients who entered the LTE period and received $\geq 1$ dose of SC ABA in the LTE regardless of the randomized treatment they received during the DB period. Safety data are presented as frequencies and IR per 6-month period over the LTE period. Incidence rate (IR; event/100 patient-yrs of exposure; 95\% CI) were calculated as events per $100 \mathrm{PY}$ of

Personal non-commercial use only. The Journal of Rheumatology Copyright @ 2018. All rights reserved. 
exposure, with the duration of exposure ending at the time of first AE occurrence. The immunogenicity analysis population included all patients who received $\geq 1$ dose of SC ABA and had immunogenicity samples collected and analyzed during the LTE period. Efficacy analyses were descriptive because they were based on as-observed data and no formal statistical tests were performed.

\section{RESULTS}

Patient disposition, baseline characteristics, and $A B A$ exposure. Of the 1385 patients who completed the DB period, $1372(99.1 \%)$ entered the LTE period and received SC ABA $125 \mathrm{mg} /$ week. One patient who discontinued during the DB period was incorrectly reported as having received SC ABA in the LTE period and was included in the "all-treated patients" analysis population $(\mathrm{n}=1373)^{5}$. A total of 945 (68.8\%) patients completed the trial. The most common reasons for discontinuation were $\mathrm{AE}(\mathrm{n}=100,7.3 \%)$, lack of efficacy $(n=89,6.5 \%)$, and withdrawal of consent $(n=81$, $5.9 \%$; Figure 1).

Demographic data and clinical characteristics at baseline for patients treated in the LTE period were similar to those observed in the DB period $^{10}: 74.5 \%$ white, $82.4 \%$ female, mean age of 49.7 years, and RA mean duration of 7.6 years. At entry into the LTE period, $99.1 \%$ of patients were receiving $\geq 15 \mathrm{mg} /$ week MTX (99.9\% orally) and $<1 \%$ a concomitant non-MTX DMARD. At each study visit during the LTE period, about one-third of patients (30-35\%) were receiving concomitant MTX at a dose of $>15 \mathrm{mg} /$ week (mean weekly dose range 15.2-16.2 mg). The remaining patients received concomitant MTX at a lower dose and $97(7.1 \%)$ patients discontinued MTX during the LTE period. During the LTE period, about $10 \%$ of patients had other DMARD, such as sulfasalazine, chloroquine, hydroxychloroquine, or azathioprine, added to their treatment. At entry into the LTE period, $68.6 \%$ of patients were receiving corticosteroids; prednisolone (705 patients, $51.3 \%$ ) was the most commonly used and throughout the study, the mean daily dose of corticosteroids remained stable (7.9 mg; SD 8.4).

Among patients who entered and received treatment in the LTE period, mean (SD; range) cumulative exposure to ABA (either formulation; combined DB and LTE periods) was 51.8 (16.5; 7-77) months for IV plus SC ABA, and 49.0 (16.6; 3-73) months for SC ABA alone. During the LTE period alone, patients received a mean (SD; range) of 186.9 (69.3; $1-284)$ SC ABA injections. Most patients $(n=1038,75.6 \%)$ missed fewer than $5 \mathrm{SC}$ injections (consecutive or nonconsecutive) during the LTE period; the median (range) number of missed injections was $1.0(0-38)$.

Safety. A total of 41 patients $(3.0 \%)$ died during the LTE period and the IR for deaths $[0.75$ (95\% CI $0.53-1.03)$

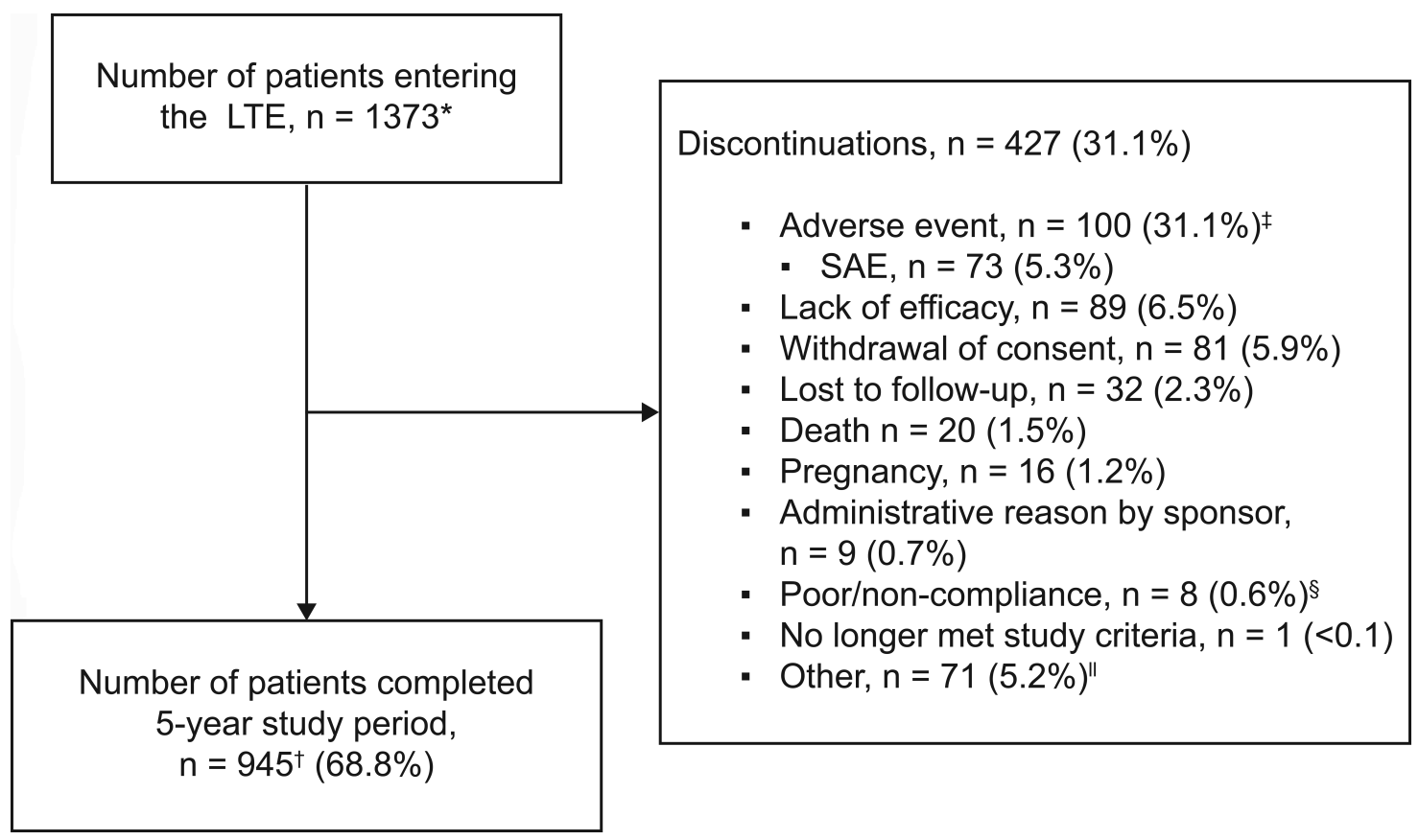

Figure 1. Patient disposition and reasons for discontinuation during the 5-year LTE period. $* 1372$ patients continued into the LTE period and received SC ABA, 1 patient was incorrectly reported to have received SC ABA and therefore included in all-treated patient population for baseline, safety, and efficacy analyses. ${ }^{\dagger} 1$ patient $(<0.1 \%)$ ongoing. ${ }^{\ddagger}$ Includes 3 patients excluded from summary of adverse events leading to discontinuation. ${ }^{\S}$ Excluded from efficacy summaries. "Including patient's decision, withdrawal of finance, clinic closure, patient move, planned surgery, pregnancy planning, need for an additional DMARD, and stopping in error before study ended. SC: subcutaneous; ABA: abatacept; LTE: longterm extension; SAE: serious adverse event; DMARD: disease-modifying antirheumatic drug.

Personal non-commercial use only. The Journal of Rheumatology Copyright @ 2018. All rights reserved. 
events/100 PY based on 5200.59 PY of exposure to SC ABA] did not increase over the study duration. The most common events leading to death were pneumonia, acute myocardial infarction, and lung cancer (including adenosquamous cell lung cancer, small cell lung cancer, metastatic lung cancer, and non-small cell lung cancer), road traffic accident, and respiratory failure (Supplementary Table 1, available with the online version of this article).

In the 5-year LTE period, 1240 (90.3\%) patients had an AE (Table 1), of which $963(77.7 \%)$ were mild or moderate, and $97(7.1 \%)$ patients discontinued treatment because of an AE. A total of $353(25.7 \%)$ patients had an SAE, with the most common being infections and infestations $(\mathrm{n}=85,6.2 \%)$. The SAE IR (event/100 PY) decreased from 9.02 (95\% CI $6.31-12.90 ; 332.6 \mathrm{PY})$ in the DB period and $8.76(95 \% \mathrm{CI}$ $7.71-9.95 ; 2717.11 \mathrm{PY})$ in the 3 -year LTE period to 7.73 (95\% CI 6.96-8.58; 4566.2 PY) during the 5-year LTE period.

Serious infections were reported in $85(6.2 \%)$ patients (Table 2), of whom 16 (1.2\%) discontinued from the LTE period. The most frequently reported (by $>1 \%$ of patients) serious infection was pneumonia in $16(1.2 \%)$ patients. Five opportunistic infections were reported in 5 patients: 1 each of peritoneal tuberculosis (which led to discontinuation), fungal esophagitis (mild), fungal eye infection (mild), fungal sinusitis (serious), and Candida infection (moderate). The infection and serious infection IR $(95 \% \mathrm{CI})$ were 38.60 (36.24-41.12) and 1.68 (1.35-2.07), respectively.

Malignancies were reported in $56(4.1 \%)$ patients in the LTE period; the malignancy IR $(95 \% \mathrm{CI})$ was 1.09 (0.84-1.42; Table 2). The most commonly reported malignancies included basal cell carcinoma (15 patients); breast cancer, squamous cell carcinoma, and squamous cell carcinoma of skin (4 patients each); thyroid neoplasm (3 patients); cervical carcinoma stage 0 , invasive ductal breast carcinoma, non-small cell lung cancer, and prostate cancer (2 patients each). All other malignancies occurred in 1 patient each.

Autoimmune disorders (prespecified) were reported in 67 (4.9\%) patients with an IR (95\% CI) of 1.33 (1.05-1.69; Table 2). The reported autoimmune disorders included psoriasis (13 patients), chronic gastritis (11 patients), Sjögren syndrome (10 patients), and vasculitis (6 patients). Serious autoimmune disorders were reported in 7 patients ( 2 vasculitis, 1 demyelinating polyneuropathy, 1 multiple sclerosis, 1 uveitis, 1 ulcerative colitis, and 1 psoriasis), and 5 patients (1 multiple sclerosis, 2 vasculitis, and 2 ulcerative colitis) discontinued because of an autoimmune disorder.

Systemic injection reactions (nonlocal AE that occurred during the first $24 \mathrm{~h}$ after SC ABA injection) were reported in $161(11.7 \%)$ patients in the LTE period and at a similar

Table 1. Summary of AE reported in the 5-year study period.

\begin{tabular}{|c|c|c|c|c|c|c|c|c|}
\hline \multirow[t]{2}{*}{$\mathrm{AE}$} & \multicolumn{2}{|c|}{$\begin{array}{c}\text { DB 24-week Period SC ABA + } \\
\text { MTX, } n=744\end{array}$} & \multicolumn{2}{|c|}{$\begin{array}{c}\text { DB 24-week Period IV ABA } \\
+ \text { MTX, } n=731\end{array}$} & \multicolumn{2}{|c|}{$\begin{array}{c}\text { LTE 174-week Period } \\
\text { SC ABA + MTX, } n=1372\end{array}$} & \multicolumn{2}{|c|}{$\begin{array}{l}\text { LTE 5-year Period SC } \\
\text { ABA + MTX, } n=1373\end{array}$} \\
\hline & $\mathrm{n}(\%)$ & IR $(95 \%$ CI $)$ & $\mathrm{n}(\%)$ & IR $(95 \% \mathrm{CI})$ & $\mathrm{n}(\%)$ & IR $(95 \%$ CI $)$ & $\mathrm{n}(\%)$ & IR $(95 \%$ CI $)$ \\
\hline SAE & $31(4.2)$ & $9.02(6.31-12.90)$ & $36(4.9)$ & $11.14(8.04-15.44)$ & $238(17.3)$ & $8.76(7.71-9.95)$ & $353(25.7)$ & $7.73(6.96-8.58)$ \\
\hline Death & $1(0.1)$ & $0.29(0.01-1.64)$ & $5(0.7)$ & $1.51(0.49-3.53)$ & $21(1.5)$ & $0.71(0.44-1.09)$ & $41(3.0)$ & $0.75(0.53-1.03)$ \\
\hline $\begin{array}{l}\text { AE leading to } \\
\text { discontinuation }\end{array}$ & $16(2.2)$ & $4.75(2.72-7.72)$ & $25(3.4)$ & $7.62(4.93-11.26)$ & $56(4.1)$ & $1.90(1.43-2.47)$ & $97(7.1)$ & $1.87(1.52-2.28)$ \\
\hline
\end{tabular}

Includes data through 56 days after the last dose date of the LTE period. Includes all deaths reported during the LTE period including those that occurred $>56$ days after the last dose date, regardless of timing of death. AE: adverse event; DB: double-blind; ABA: abatacept; IR: incidence rate (events per 100 patient-yrs); IV: intravenous; LTE: longterm extension; MTX: methotrexate; SAE: serious AE; SC: subcutaneous.

Table 2. AE of special interest reported during the 5-year study period.

\begin{tabular}{|c|c|c|c|c|c|c|c|c|}
\hline \multirow[t]{2}{*}{$\mathrm{AE}$} & \multicolumn{2}{|c|}{$\begin{array}{c}\text { DB 24-week Period SC } \\
\text { ABA + MTX, } n=744\end{array}$} & \multicolumn{2}{|c|}{$\begin{array}{l}\text { DB 24-week Period IV } \\
\text { ABA + MTX, n = } 731\end{array}$} & \multicolumn{2}{|c|}{$\begin{array}{l}\text { LTE 174-week Period SC } \\
\text { ABA + MTX, } n=1372\end{array}$} & \multicolumn{2}{|c|}{$\begin{array}{c}\text { LTE 5-year Period SC } \\
\text { ABA + MTX, } \mathrm{n}=1373^{*}\end{array}$} \\
\hline & $\mathrm{n}(\%)$ & IR $(95 \% \mathrm{CI})$ & $\mathrm{n}(\%)$ & IR $(95 \% \mathrm{CI})$ & $\mathrm{n}(\%)$ & IR $(95 \% \mathrm{CI})$ & $\mathrm{n}(\%)$ & IR $(95 \% \mathrm{CI})$ \\
\hline Serious infections & $5(0.7)$ & $1.48(0.62-3.56)$ & $10(1.4)$ & $3.05(1.64-5.67)$ & $50(3.6)$ & $1.72(1.30-2.27)$ & $85(6.2)^{\dagger}$ & $1.68(1.35-2.07)$ \\
\hline Autoimmune & & & & & & & & \\
\hline disorders & $6(0.8)$ & $1.78(0.80-3.96)$ & $6(0.8)$ & $1.83(0.82-4.07)$ & $38(2.8)$ & $1.31(0.95-1.79)$ & $67(4.9)^{\ddagger}$ & $1.33(1.05-1.69)$ \\
\hline
\end{tabular}

* At entry into the LTE period, $99.1 \%$ of patients were taking MTX and $<1 \%$ were taking a concomitant non-MTX DMARD. ${ }^{\dagger}$ Including pneumonia $(\mathrm{n}=16)$, urinary tract infection $(n=6)$, and appendicitis $(n=5) .{ }^{\ddagger}$ Including psoriasis $(n=13)$, chronic gastritis $(n=11)$, Sjögren syndrome $(n=10)$, and vasculitis $(n=6) . ~{ }^{\S}$ Including basal cell carcinoma $(n=15)$; breast cancer, squamous cell carcinoma, squamous cell carcinoma of skin $(n=4$ each); thyroid neoplasm $(\mathrm{n}=3)$; and cervical carcinoma Stage 0, invasive ductal breast carcinoma, non-small cell lung cancer, prostate cancer $(\mathrm{n}=2$ each). DB: double blind; DMARD: disease-modifying antirheumatic drug; IR: incidence rate (events per 100 patient-yrs); IV: intravenous; LTE: longterm extension; MTX: methotrexate; SAE: serious adverse event; SC: subcutaneous; ABA: abatacept. 
frequency to that observed in patients who received SC ABA in the DB period (56/736 patients, $7.6 \%)$. These were mostly mild and moderate in severity, except in 4 patients ( 2 serious chest pain, 1 serious headache, and 1 serious hypertension), and rarely resulted in discontinuation of study drug (1 event: headache). No cases of anaphylaxis were reported in the LTE period and no notable safety issues emerged from the evaluation of laboratory or vital signs data.

Local injection-site reactions (defined as prespecified $\mathrm{AE}$ that occurred at the site of SC ABA injection) were reported in $33(2.4 \%)$ patients and were mostly mild in intensity. There were 2 events (injection-site nodule and injection-site hemorrhage) that were moderate, and 2 injection-site reactions (erythema and pruritus, both mild in intensity) in 1 patient that led to early discontinuation.

IR of $\mathrm{AE}$ of special interest remained stable with increasing duration of exposure during the LTE period and are shown by 6-month intervals in Figure 2, and Supplementary Figure 1, and Supplementary Table 2 (available with the online version of this article).

Immunogenicity. Immunogenicity was assessed in 1365 patients; during the LTE period, a total of 316 (23.2\%) patients were positive for anti-ABA antibodies (patients positive for both CTLA-4 and possibly Ig, and Ig and/or junction region were counted only once; Table 3 ). A total of $229(16.8 \%)$ patients had an ABA-induced positive antibody response with specificity for CTLA-4 and possibly Ig, and $142(10.4 \%)$ for Ig and/or junction region. Among the 229 patients who were positive for antibodies toward the CTLA-4 and possibly Ig portion during treatment $(\mathrm{n}=123)$ and posttreatment periods $(n=142)$, samples from 132 patients met the criteria for further testing for neutralizing antibody activity. Of those further tested, 61 (46.2\%) patients had $\geq 1$ sample that was positive for neutralizing antibody, mostly occurring following the last study drug dose. The exposure-based IR (95\% CI) of on-treatment positive ABA antibody response was $4.58(3.98-5.24)$ per 100 PY of exposure across the entire 5-year LTE period, with 2.56 (2.13-3.06) toward CTLA-4 and possibly Ig, and 2.52 (2.10-3.01) toward Ig and/or junction region. There was no increase in the 6-monthly IR over time. The immunogenic profile following the discontinuation of treatment was not
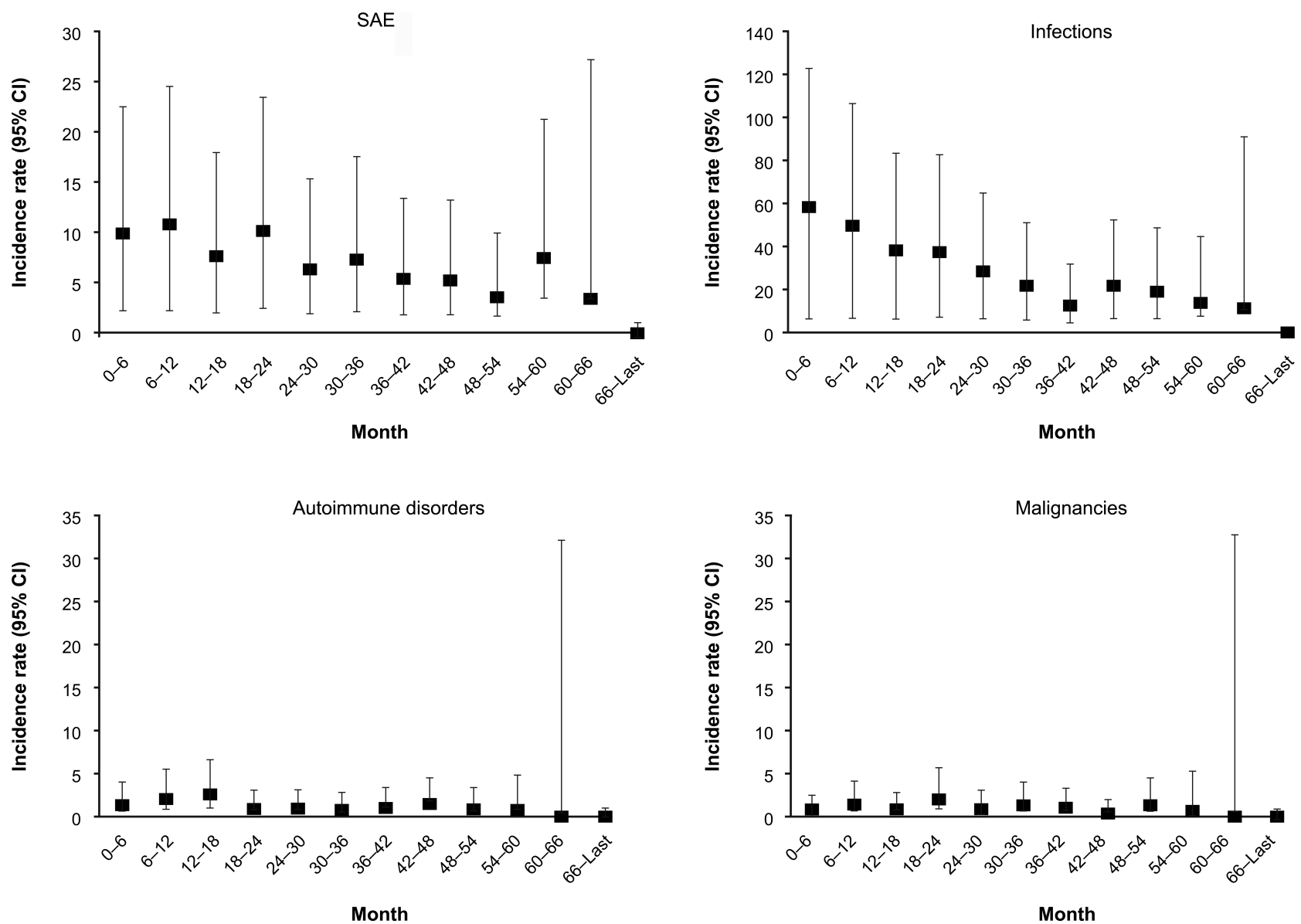

Figure 2. IR of AE of special interest (SAE, infections, autoimmune disorders, and malignancies) by 6-month intervals over the 5-year LTE period. IR: incidence rate (event/100 patient-yrs of exposure); AE: adverse events; SAE: serious AE; LTE: longterm extension.

Personal non-commercial use only. The Journal of Rheumatology Copyright $\odot$ 2018. All rights reserved. 
Table 3. Immunogenicity rates over the 5-year LTE period. Values are $\mathrm{n} / \mathrm{N}(\%)$ unless otherwise specified.

\begin{tabular}{lcc}
\hline Immunogenicity Category & CTLA-4 and Possibly Ig & Ig and/or Junction Region \\
\hline Any positive response, including posttreatment & $229 / 1365(16.8)$ & $142 / 1365(10.4)$ \\
Positive response while receiving treatment & $123 / 1358(9.1)$ & $122 / 1358(9.0)$ \\
Positive response posttreatment* & $142 / 1111(12.8)$ & $69 / 1111(6.2)$ \\
IR (95\% CI) while receiving treatment & $2.56(2.13-3.06)$ & $2.52(2.10-3.01)$ \\
Persistent response while receiving treatment** & $19 / 1330(1.4)$ & $47 / 1330(3.5)$ \\
Neutralizing antibody response while receiving treatment & $61 / 1358(4.5)$ & $\mathrm{NA}$ \\
\hline
\end{tabular}

$* 28,85$, or 168 days after the discontinuation of study drug. $* * 2$ consecutive positive responses $\geq 12$ weeks apart. CTLA-4: T cell lymphocyte costimulation inhibitors; Ig: immunoglobulin; LTE: longterm extension; NA: not applicable; IR: incidence rate (events per 100 patient-yrs).

different from the profile observed during the period on treatment.

As during the DB and 3-year LTE period ${ }^{10,13}$, the presence of a positive antibody seroconversion response did not appear to affect the efficacy (including ACR20, 50, and 70, DAS28-CRP $\leq 3.2$, DAS28-CRP $<2.6$, and HAQ-DI) or safety of ABA during the 5-year LTE period of our study. A review of the ACR20 responses in patients with anti-ABA neutralizing antibodies did not reveal any trends toward lessening efficacy following the occurrence of neutralizing antibodies (data not shown), consistent with the immunogenicity results found at 3 years ${ }^{10}$. Further, to determine whether the ACR20 response shifted from positive to negative following a positive antibody response, the antibody response for patients with persistent positive antibodies was evaluated. In general, there was no association between positive immunogenicity and a loss of ACR 20 response. Among the 4 patients who had a persistent antibody response and a shift from positive to negative ACR20 response, none had neutralizing antibodies.

The antibody responses were reviewed for patients who experienced local and systemic injection reactions and autoimmune disorders, and no consistent patterns between injection reactions or medically important $\mathrm{AE}$ and positive antibody responses were identified. Among the 33 patients who reported a prespecified local injection-site reaction during the LTE period, only 3 had a positive immunogenicity response (no association between event timing and positive response was found).

Of the 161 patients who reported prespecified systemic injection reactions, 32 had $\geq 1$ positive immunogenicity response. Of those who had prespecified autoimmune disorders, 11 had a positive immunogenicity response.

Efficacy. Efficacy in the LTE period was consistent with that observed in the DB period and was maintained over the study period in patients who remained on the study drug (Table 4). As-observed ACR20, 50, and 70 responses at Day 169 were $80.1 \%(1087 / 1357), 53.2 \%$ (724/1362), and $27.2 \%$ (371/ $1362)$, and at Day 1821 were $84.6 \%$ (356/421), $65.5 \%$ (277/ $423)$, and $44.9 \%$ (191/425), respectively. The beneficial effects of ABA on physical function (HAQ-DI response), disease activity, and disease remission observed at the end of the DB period were also maintained throughout the 5-year LTE period. The mean percentage improvement in HAQ-DI score at Day 169 was $42.9 \%$ (95\% CI 40.7-45.1) and at Day 1821 was $45.4 \%$ (95\% CI 40.7-50.1). At Day 169, 24.6\% $(\mathrm{n}=334)$ of patients had DAS28-CRP $<2.6$ and $40.8 \%$ $(\mathrm{n}=553)$ had DAS28-CRP $\leq 3.2$; at Day $1821,40.9 \%$ $(\mathrm{n}=169)$ of patients had DAS28-CRP $<2.6$ and $57.6 \%$ $(\mathrm{n}=238)$ had DAS28-CRP $\leq 3.2$ (Table 4; as-observed analysis). Of 334 patients who had DAS28-CRP $<2.6$ at Day $169,67.3 \%$ (37/55 patients with data available at Day 1821) maintained DAS28-CRP $<2.6$ at Day 1821. Of 553 patients who had DAS28-CRP $\leq 3.2$ at Day $169,76.2 \%(64 / 84$ patients with data available at Day 1821) maintained DAS28-CRP $\leq 3.2$ at Day 1821. Improvements in pain and patient global assessment scores were also seen throughout the trial (data not shown).

Pharmacokinetics. ABA trough concentrations were higher following SC administration compared with IV and remained consistent within both groups from days 85 to 169 (DB period). ABA concentrations remained stable (geometric mean minimum plasma concentration at steady state range 25.1-28.8 $\mu \mathrm{g} / \mathrm{ml}$, days 253-729) over the LTE period. In addition, ABA trough concentrations were not affected by an anti-ABA antibody response in the LTE period.

\section{DISCUSSION}

During the 5-year LTE period of the ACQUIRE study, the safety and efficacy of SC ABA $125 \mathrm{mg} /$ week were consistent with those seen in the initial 6-month DB period and previously reported 3-year followup, and with the established longterm safety profile of IV $\mathrm{ABA}^{10,12,13}$. These data, including the observation that no new safety signals emerged during this LTE period, demonstrate that SC administration of ABA $125 \mathrm{mg} /$ week had a consistent safety profile and durable efficacy in longterm ( $\geq 5 \mathrm{yrs}$ ) treatment of patients with RA and an inadequate response to MTX.

The safety profile of SC ABA over the duration of the ACQUIRE study was acceptable. Most AE reported in the LTE period were mild or moderate (77.7\%). Importantly, IR of SAE and AE of special interest, including serious infec-

Personal non-commercial use only. The Journal of Rheumatology Copyright $@$ 2018. All rights reserved 
Table 4. Efficacy during the LTE period. Values are \% (n/N) $(95 \% \mathrm{CI})$.

\begin{tabular}{|c|c|c|c|c|}
\hline Endpoint & Day 169 & Day 729 & Day 1261 & Day 1821 \\
\hline \multicolumn{5}{|c|}{ No. patients, $\%(\mathrm{n} / \mathrm{N})(95 \% \mathrm{CI})$} \\
\hline ACR20 & $80.1(1087 / 1357)(78.0-82.2)$ & $82.0(973 / 1187)(79.8-84.2)$ & $84.6(904 / 1068)(82.5-86.8)$ & $84.6(356 / 421)(81.1-88.0)$ \\
\hline ACR70 & $27.2(371 / 1362)(24.9-29.6)$ & $37.4(443 / 1186)(34.6-40.1)$ & $40.9(438 / 1070)(38.0-43.9)$ & $44.9(191 / 425)(40.2-49.7)$ \\
\hline DAS $28-C R P \leq 3.2$ & $40.8(553 / 1355)(38.2-43.4)$ & $50.7(600 / 1183)(47.9-53.6)$ & $55.0(585 / 1064)(52.0-58.0)$ & $57.6(238 / 413)(52.9-62.4)$ \\
\hline DAS28-CRP $<2.6$ & $24.6(334 / 1355)(22.4-26.9)$ & $34.7(411 / 1183)(32.0-37.5)$ & $39.9(425 / 1064)(37.0-42.9)$ & $40.9(169 / 413)(36.2-45.7)$ \\
\hline \multicolumn{5}{|c|}{ Improvement from baseline, mean (SE) \% (95\% CI) } \\
\hline $\mathrm{CRP}, \mathrm{mg} / \mathrm{dl}$ & $15.04(7.80)(-0.27$ to 30.35$)$ & $9.70(11.58)(-13.03$ to 32.43$)$ & $4.21(7.90)(-11.28$ to 19.70$)$ & $-7.05(19.29)(-44.97$ to 30.88$)$ \\
\hline
\end{tabular}

ACR20: American College of Rheumatology 20\% improvement criteria; DAS28-CRP: 28-joint count Disease Activity Score using C-reactive protein; LTE: longterm extension.

tions, opportunistic infections, local injection-site reactions, systemic injection reactions, malignancies, and autoimmune disorders, remained low and stable with increased duration of exposure. The IR for deaths (0.75 events/100 PY) was stable over the study duration and is comparable with previously reported rates for $\mathrm{SC} \mathrm{ABA}^{9,12,14}$. The IR for serious infections (1.68) was similar to that previously reported for longterm IV ABA (hospitalized infections, 1.55) ${ }^{15}$ and SC ABA $(1.79)^{9}$. Similar to previous reports, there was a low rate of opportunistic infections and new tuberculosis infections or reactivation ${ }^{9}$. Further, the IR for malignancy (1.09) and autoimmune disorders (1.33) reported here were similar to those reported for patients with RA treated with IV and SC ABA over the long term (malignancy 1.32; autoimmune disorders 1.37$)^{9}$, and standardized IR estimated for patients with RA in population- and community-based cohorts (malignancy $1.06-1.13)^{2}$.

Antibody responses to biologic agents such as ABA can cause general immune-mediated toxicities (e.g., systemic infusion reactions, local injection reactions, and other hypersensitivity reactions). Hence, consideration of local injection-site reactions is important with SC administration of biologic DMARD. The frequency of local injection-site reactions with $\mathrm{ABA}$ in the ACQUIRE LTE period was low $(2.4 \%)$ and similar to that previously reported $(4.1 \%)^{16}$. There is a theoretical concern that antibodies directed to the CTLA-4 portion of ABA could react with endogenous CTLA-4 expressed on T lymphocytes, leading to neutralization and potential immunostimulatory effects and autoimmunity ${ }^{17}$. However, no increase in autoimmune disorders was observed with ABA treatment over the 5-year LTE period. As previously reported, the rate of immunogenic response was low for ABA, with no increase observed with continued dosing over the study period ${ }^{18}$. Importantly, there was no association between development of anti-ABA antibodies and ABA safety or efficacy over the 5-year period, or pharmacokinetics over the tested period through Day 729 .

The high patient retention, about $69 \%$ completing $\geq 5$ years of treatment, confirms efficacy and safety profile of
ABA; overall, only $6.5 \%$ of patients discontinued because of lack of efficacy and $7.3 \%$ because of an AE.

There are limitations to our study that must be considered, including the potential bias toward improved efficacy outcomes in open-label, uncontrolled, longterm studies. Efficacy was an as-observed completer analysis, and does not record discontinuations resulting from loss of efficacy, safety, or other reasons, or any relationship between concomitant drug use and clinical response. In addition, the LTE period continued until the SC formulation became commercially available and thus participation in the LTE period varied among patients based on their geographic location. Despite these limitations, the 5-year longterm data from this global trial provide information on outcomes that would be expected in clinical practice with longterm ABA treatment.

The longterm safety and efficacy data in this trial are consistent with previously published data for the $\mathrm{IV}^{4,5,7,8}$ and $\mathrm{SC}^{9,10,11,12,13}$ formulations of $\mathrm{ABA}$, and demonstrate that longterm SC ABA use has a consistent safety profile and durable efficacy in patients with RA who continue to receive treatment.

\section{ACKNOWLEDGMENT}

The authors thank Zoe Tzogas of Bristol-Myers Squibb for performing immunogenicity analyses. Zoe Tzogas was not involved in study conception and design, or analysis and interpretation of data. Professional medical writing and editorial assistance was provided by Fiona Boswell, $\mathrm{PhD}$, at Caudex and was funded by Bristol-Myers Squibb.

\section{ONLINE SUPPLEMENT}

Supplementary material accompanies the online version of this article.

\section{REFERENCES}

1. Smitten AL, Simon TA, Hochberg MC, Suissa S. A meta-analysis of the incidence of malignancy in adult patients with rheumatoid arthritis. Arthritis Res Ther 2008;10:R45.

2. Simon TA, Thompson A, Gandhi KK, Hochberg MC, Suissa S. Incidence of malignancy in adult patients with rheumatoid arthritis: a meta-analysis. Arthritis Res Ther 2015;17:212.

3. Westhovens R, Kremer JM, Moreland LW, Emery P, Russell AS, Li $\mathrm{T}$, et al. Safety and efficacy of the selective costimulation modulator

Personal non-commercial use only. The Journal of Rheumatology Copyright $\odot$ 2018. All rights reserved. 
abatacept in patients with rheumatoid arthritis receiving background methotrexate: a 5-year extended phase IIB study. J Rheumatol 2009;36:736-42.

4. Westhovens R, Kremer J, Emery P, Russell A, Alten R, Barre E, et al. Long-term safety and efficacy of abatacept in patients with rheumatoid arthritis and an inadequate response to methotrexate: a 7-year extended study. Clin Exp Rheumatol 2014;32:553-62.

5. Takeuchi T, Matsubara T, Urata Y, Suematsu E, Ohta S, Honjo S, et al; Japan Abatacept Study Group. Phase III, multicenter, open-label, long-term study of the safety of abatacept in Japanese patients with rheumatoid arthritis and an inadequate response to conventional or biologic disease-modifying antirheumatic drugs. Mod Rheumatol 2014;24:744-53.

6. Kremer JM, Russell AS, Emery P, Abud-Mendoza C, Szechinski J, Westhovens R, et al. Long-term safety, efficacy and inhibition of radiographic progression with abatacept treatment in patients with rheumatoid arthritis and an inadequate response to methotrexate: 3-year results from the AIM trial. Ann Rheum Dis 2011;70:1826-30.

7. Kremer JM, Peterfy C, Russell AS, Emery P, Abud-Mendoza C, Sibilia J, et al. Longterm safety, efficacy, and inhibition of structural damage progression over 5 years of treatment with abatacept in patients with rheumatoid arthritis in the abatacept in inadequate responders to methotrexate trial. J Rheumatol 2014;41:1077-87.

8. Genovese MC, Schiff M, Luggen M, Le Bars M, Aranda R, Elegbe A, et al. Longterm safety and efficacy of abatacept through 5 years of treatment in patients with rheumatoid arthritis and an inadequate response to tumor necrosis factor inhibitor therapy. J Rheumatol 2012;39:1546-54.

9. Schiff M. Subcutaneous abatacept for the treatment of rheumatoid arthritis. Rheumatology 2013;52:986-97.

10. Genovese MC, Pacheco-Tena C, Covarrubias A, Leon G, Mysler E, Keiserman M, et al. Subcutaneous abatacept for the treatment of rheumatoid arthritis: longterm data from the ACQUIRE trial. J Rheumatol 2014;41:629-39.

11. Amano K, Matsubara T, Tanaka T, Inoue H, Iwahashi M, Kanamono T, et al; Japan Abatacept Study Group. Long-term safety and efficacy of treatment with subcutaneous abatacept in Japanese patients with rheumatoid arthritis who are methotrexate inadequate responders. Mod Rheumatol 2015;25:665-71.

12. Alten R, Kaine J, Keystone E, Nash P, Delaet I, Genovese MC. Long-term safety of subcutaneous abatacept in rheumatoid arthritis: integrated analysis of clinical trial data representing more than four years of treatment. Arthritis Rheumatol 2014;66:1987-97.

13. Genovese MC, Covarrubias A, Leon G, Mysler E, Keiserman M, Valente R, et al. Subcutaneous abatacept versus intravenous abatacept: a phase IIIb noninferiority study in patients with an inadequate response to methotrexate. Arthritis Rheum 2011;63:2854-64.

14. Lunt M, Watson KD, Dixon WG, British Society for Rheumatology Biologics Register Control Centre Consortium, Symmons DP, Hyrich KL; British Society for Rheumatology Biologics Register. No evidence of association between anti-tumor necrosis factor treatment and mortality in patients with rheumatoid arthritis: results from the British Society for Rheumatology Biologics Register. Arthritis Rheum 2010;62:3145-53.

15. Weinblatt ME, Moreland LW, Westhovens R, Cohen RB, Kelly SM, Khan N, et al. Safety of abatacept administered intravenously in treatment of rheumatoid arthritis: integrated analyses of up to 8 years of treatment from the abatacept clinical trial program. J Rheumatol 2013;40:787-97.

16. Schiff M, Weinblatt ME, Valente R, van der Heijde D, Citera G, Elegbe A, et al. Head-to-head comparison of subcutaneous abatacept versus adalimumab for rheumatoid arthritis: two-year efficacy and safety findings from AMPLE trial. Ann Rheum Dis 2014;73:86-94.

17. Schellekens H. The immunogenicity of therapeutic proteins. Discov Med 2010;9:560-4.

18. Kaine J, Gladstein G, Strusberg I, Robles M, Louw I, Gujrathi S, et al. Evaluation of abatacept administered subcutaneously in adults with active rheumatoid arthritis: impact of withdrawal and reintroduction on immunogenicity, efficacy and safety (phase IIIb ALLOW study). Ann Rheum Dis 2012;71:38-44. 